International Journal of Wireless \& Mobile Networks (IJWMN) Vol. 4, No. 1, February 2012

\title{
EFFICIENT SYMBOL ERROR RATE ANALYSIS OF COOPERATIVE NON-REGENERATIVE RELAY SYSTEMS OVER GENERALIZED FADING CHANNELS
}

\author{
Oluwatobi Olabiyi $^{1}$ and Annamalai Annamalai ${ }^{2}$ \\ Centre of Excellence for Communication Systems Technology Research \\ Department of Electrical and Computer Engineering \\ Prairie View A\&M University, Texas 77446 \\ 1engr3osegmail.com ${ }^{2}$ aaannamalai@pvamu.edu
}

\begin{abstract}
In this article, we develop a unified approach to the asymptotic performance analysis of symbol error rates for two-hop cooperative non-regenerative multi-relay networks over a myriad of stochastic channel models. Our proposed moment generating function (MGF) based analytical framework is also sufficiently general to encapsulate all types of coherent, differentially-coherent and non-coherent digital modulation schemes. In addition to simplifying the computation complexity of average symbol error rate (ASER) for cooperative amplify-and-forward (CAF) diversity systems treated in the literature, we also provide an accurate approximation for the desired ASER in a number of fading environments that heretofore had resisted a simple solution. The accuracy of our approximations has been validated with the closed-form formulas that are available for specific fading environments (e.g., Rayleigh and Nakagami-m channels) and via computer (Monte Carlo) simulations. Our analytical approach may also be extended to other types of cooperative strategies and multi-hop networks, although it is beyond the scope of this article.
\end{abstract}

\section{KEYWORDS}

Asymptotic analysis, cooperative diversity systems, MGF method, amplify-and-forward relay networks

\section{INTRODUCTION}

The evaluation of ASER performance for both coded and uncoded wireless transmissions over fading channels is often carried out in two steps: First, an exact or approximate formula for the conditional error probability (i.e., error rate performance in a fixed channel realization perturbed by additive white Gaussian noise) is obtained, which is a function of both the random received signal-to-noise-ratio (SNR) $\gamma$ and the choice of modulation/detection scheme. In the second step, the conditional error probability (CEP) is averaged over the probability density function (PDF) of the SNR for a specified fading environment, viz.,

$$
P_{s}(\varepsilon)=\int_{0}^{\infty} P_{s}(\varepsilon \mid \gamma) f_{\gamma}(\gamma ; \Omega, h) d \gamma
$$

where $\Omega=E[\gamma]$ corresponds to the mean link SNR and $h$ is the fading shape parameter associated with the fading density function $f_{\gamma}(\gamma ; \Omega, h)$.

In general, efficient evaluation of the averaging problem described in (1) can be carried out via one of the four cases listed below:

- Case I: Use exact expressions for both the CEP $P_{s}(\varepsilon \mid \gamma)$ and $\operatorname{PDF} f_{\gamma}(\gamma ; \Omega, h)$;

- Case II: Employ an approximate or asymptotic expression for the CEP $P_{s}(\varepsilon \mid \gamma)$ but exact expression for the PDF $f_{\gamma}(\gamma ; \Omega, h)$; 
International Journal of Wireless \& Mobile Networks (IJWMN) Vol. 4, No. 1, February 2012

- Case III: Employ an exact expression for $P_{s}(\varepsilon \mid \gamma)$ but use asymptotic expression or bounds for the PDF $f_{\gamma}(\gamma ; \Omega, h)$;

- Case IV: Employ approximate, asymptotic or bounds for both the $\operatorname{CEP} P_{s}(\varepsilon \mid \gamma)$ and $\operatorname{PDF}$ $f_{\gamma}(\gamma ; \Omega, h)$.

Although the results obtained via Case I and Case II can be highly accurate, the application of such approaches for ASER analysis of CAF diversity systems is a daunting task, which can be attributed to the difficulty associated with the determination of the exact $\operatorname{PDF} f_{\gamma}(\gamma ; \Omega, h)$ in a generalized fading environment. For this reason, most of the existing "exact" results for cooperative non-regenerative diversity systems were limited to either Rayleigh [1]-[3] or Nakagami-m channels with independent and identically distributed (i.i.d) diversity paths [4]. However, this may not be a good representative of the practical operating environments since signal transmission over distinct wireless links may experience different amount of fading and undergo different propagation paths before arriving at the destination node. In [5], Ribeiro et. al. developed an asymptotic expression for CAF diversity systems that employ BPSK modulation in Rayleigh and Rice fading channels (although all their results were limited to only Rayleigh fading) using an asymptotic analysis technique similar to that developed in [6] for noncooperative diversity systems. The key idea here is to approximate the PDF of each of the independent wireless links as $t_{1} \gamma^{t_{2}}$ (i.e., Case III), where $t_{1}$ and $t_{2}$ are parameters specific to the chosen stochastic channel model. Whereas in [7], Ma et. al. extended the analysis in [6] to a wide range of digital modulation schemes that employ a generalized selection combining diversity receiver (but for non-cooperative network). In [2] and [8]-[11], other researchers have developed bounds for the MGF of end-to-end SNR of CAF in Rayleigh [2][8], Nakagami-m [9][10] and Rice [11] fading environments (i.e., Case III).

In this article, we develop a new asymptotic expression for the ASER of CAF diversity systems by approximating the CEP in a "desirable exponential form" (i.e., $P_{s}(\varepsilon \mid \gamma) \approx a e^{-b \gamma}$ ) in conjunction with an asymptotic approximation of the PDF of SNR of the relayed diversity path (Case IV). The resulting closed-form ASER formulas are applicable to wide range of digital modulation schemes (i.e., not restricted to BPSK as in [5]) and fading environments (including mixed-fading scenario). Interestingly, our results are much more general and more accurate especially in less severely faded channel conditions than the corresponding formulas presented in [5] yet preserves the low-computational complexity for evaluating the ASER (i.e., the final ASER formula is in closed-form), which in turn may facilitate system level optimization (e.g., optimal power assignment problem in cooperative relay systems, joint-design of link-adaptive CAF networks, etc.).

\section{RELATED WORK}

The new paradigm of cooperative wireless communication has revolutionized the design of emerging wireless networks by exploiting the broadcast nature of wireless transmissions and the inherent spatial diversity in wireless networks (i.e., spatially separated communication nodes in cluster). In particular, this novel communication protocol has the potential to overcome the practical implementation issue of packing a large number of antenna elements in small-sized handheld or portable devices. It is also important to highlight that the classical implementation of multiple-input-multiple-output (MIMO) antenna array to reap the benefits of spatial diversity and/or multiplexing gain requires mobile devices that are currently equipped with a single antenna element to be replaced with an antenna array (to take advantage of higher data rate and reduced transmission error offered by the MIMO technology). With the advent of cooperative communications, such costly changes are no longer necessary as multiple communication nodes in a cluster may collaborate and mimic the operation of a MIMO system with "distributed 
International Journal of Wireless \& Mobile Networks (IJWMN) Vol. 4, No. 1, February 2012

antenna elements" (i.e., virtual or synthetic MIMO). In other words, cooperative relaying strategies facilitate an evolutionary path for harnessing the benefits of MIMO technology with existing small form-factor hand-held devices that are not equipped with an antenna array (e.g., cell-phones). Besides, the relay nodes afford the sender to communicate to the destination node with reduced transmitter power, and as such it is environmentally friendly (i.e., green communications) and may also reduce the security concerns in tactical wireless networks by ensuring a low probability of detection and interception of the transmitted waveform. Cooperative relaying architecture also offers a modular and flexible solution to meet a prescribed design objective (e.g., data rate, error rate, energy constraint, etc.) and quality of service assurance and thus, it is very attractive for a wide range of wireless applications including battlefield communications, first-responder and disaster management networks, cellular communications, wireless sensor networks, vehicular/mobile ad-hoc networks, satellite and deep-space communications, among many others.

The concept of relay communication itself is as old one, but recent advances in digital signal processing have made the implementation of various cooperative relaying protocols feasible. The introduction of the practical cooperative relaying protocols by Laneman et. al. [12], [16] has resulted in a surge of research interests in field of wireless cooperative relaying. Over the last eight years, many contributions have been made on the design, implementation and performance analysis of cooperative diversity systems. In general, cooperative diversity protocols can be classified into one of three main categories, namely decode-and-forward, amplify-and-forward and compress-and-forward protocols [12], [16]-[20]. In the decode-andforward (DF) relaying protocol, the relay nodes decode the transmitted information and forward only correctly received information towards the destination. This protocol requires the relay to have same processing capability as the receiver, and therefore increases the delay and complexity of the relay nodes. In the compressed-and-forward technique, the relay only retransmits the quantized version of the source-transmitted signal. Based on the available channel state information (CSI), the relay re-encodes the signal to match the prevailing channel condition and the destination can further exploit the diversity from different encoding schemes of signal employed by source-destination and relay-to-destination to achieve higher data rate and more reliable communication. In the amplify and-forward protocol, the complexity of the relay node can be dramatically reduced as it only need to amplify the received signal and forwards it to the destination node. Since the relay does not decode the received signal, it reduces the security risk in case the relay node is compromised. However, in this protocol the error in source-to-relay message is also amplified and transmitted to the destination which increases the overall end-to-end error probability. Other variations of cooperative relaying strategies include opportunistic, incremental, variable-gain and fixed-gain (either blind or semiblind) relaying schemes that are based on the availability of CSI and the number of active participating nodes for information relaying [12], [14]-[20]. In this article, we primarily focus on variable-gain cooperative amplify-and-forward (CAF) relaying strategy although the analysis may be extended to other categories and variations of cooperative relaying strategies.

The literature dealing with the performance analyses of cooperative relay networks are quite extensive that focus on various performance metrics such as average symbol or bit error rates, ergodic/outage capacity and ergodic and outage capacity (see [1]-[12] and [14]-[34], and references therein). The performance analysis of DF relaying is relatively more mathematically tractable than that of CAF relaying (e.g., [21]-[27]). However, the analysis of CAF relay can be slightly more involved due to the difficulty in obtaining a tractable statistics of the instantaneous total received SNR at the destination node such as the PDF, cumulative distribution function $(\mathrm{CDF}$ ), and moment generating functions (MGF) of end-to-end signal-to-noise ratio (SNR). In fact, the exact closed-form statistics of end-to-end SNR is only known for Rayleigh and Nakagami-m fading with diversity relay path having i.i.d fading statistics [1]-[4] which may not be a representative of realistic operational and propagation environments. In order to extend the 
performance analysis to other fading environments, (e.g. Rice fading) with non-identical statistics, bounds for the PDF, CDF and MGF of end-to-end SNR have been developed [8]-[12]. The statistics of end-to-end SNR bound has been used for the computation of ASER, outage capacity, outage probability and spectral efficiency in many articles [8]-[12], [29]-[34]. However, the statistics of SNR bound is channel specific and can sometimes be complicated for certain fading channels (e.g., mixed-fading, Nakagami-q, composite-fading, etc.). Also, their application in performance analysis may not always result into closed-form solutions. To overcome this limitation, [5] introduced an asymptotic analysis approach based on the framework for non-cooperative systems outlined in [6]-[7] to develop simple closed-form expression for the ASER of CAF relay system in a Rice fading environment, although their results are only limited to a Rayleigh fading environment. This work was later extended to Nakagami-m fading channel in [14]. The asymptotic analysis although very simple, does not give accurate representation of the exact analysis especially in the low SNR regime and when the channel experience less severe fading. But the low SNR regime is of particular interest since cooperative diversity is likely to be employed to boost the system performance in this region (and not in the high-SNR regime)!

In this article, we develop a highly efficient asymptotic expression for the MGF of end-to-end SNR of CAF relay system. This expression is shown to be more accurate than the asymptotic analysis [5], [14] and even the upper and lower bound expressions [8]-[11] while closely approximating the exact MGF expression for the end-to-end SNR [1]-[14], [15]. This asymptotic MGF expression is then used to evaluate the average symbol error rate (ASER) of CAF multi-relay system using the MGF method in [13]. In order to take advantage of the expression in [13, Eq. (1.10)], we simplify the usually single integral form (in case of M-ary phase shift keying (MPSK) modulation) or $Q$-function type (in case of M-ary quadrature amplitude (MQAM) modulation) CEP expressions by expressing them in desirable exponential form using a mean square error optimization routine. Hence, our final ASER formula is only expressed as a weighted MGF function evaluated at certain arguments (corresponding to a specific digital modulation). The proposed closed-form ASER expression can be readily applied for further system-level optimization (e.g., optimum power allocation, optimum relay node placement, and cross-layer design for throughput maximization) which is of interest for optimizing the emerging mobile wireless and sensor networks.

\section{Approximate Conditional Symbol Error Probability}

In this section, we summarize the approximate CEP in a desirable exponential form $\left(P_{s}(\varepsilon \mid \gamma) \approx a e^{-b \gamma}\right)$ with constants $a$ and $b$, which are obtained using a minimum mean square error (MMSE) criterion for curve fitting of the exact CEP expressions in second column of Table 1 and Table 2. The optimization equation can be written as

$$
a^{*}, b^{*}=\min _{a, b}\left\{\frac{1}{J} \sum_{i=1}^{J}\left[\ln P_{s}\left(\varepsilon \mid \gamma_{i}\right)-\ln a+b \gamma_{i}\right]^{2}\right\}
$$

where $J$ is the number of points used in the MMSE minimization. This particular exponential form for the CEP facilitates the averaging problem over the PDF of SNR since the resulting expression is simply the MGF of SNR (i.e., Laplace transform of the PDF).

Table 1 and Table 2 summarizes the values for our optimized coefficients $a$ and $b$ of our approximate CEP formula for a wide range of coherent, differentially coherent and noncoherent digital modulation schemes. The tightness of approximate CEP formulas is depicted in Fig. 1 through Fig. 3. It is obvious that our exponential form closely approximate the exact CEP especially for MPSK and MQAM modulation schemes. In order to obtain optimized coefficients $a^{*}$ and $b^{*}$ for each modulation scheme, the curve fitting is done over a practical range of SNR. In our case, the range of SNR and the number of points for each modulation scheme with constellation size $M$ are summarized below: 
International Journal of Wireless \& Mobile Networks (IJWMN) Vol. 4, No. 1, February 2012

$M$-PSK: $[M=2,0-8 \mathrm{~dB}, J=17] ;[M=4,0-20 \mathrm{~dB}, J=41] ;[M=8,0-22 \mathrm{~dB}, J=45] ;[M=16,0-$ $30 \mathrm{~dB}, J=61] ;[M=32,0-30 \mathrm{~dB}, J=61]$

$M$-QAM: $[M=4,0-20 \mathrm{~dB}, J=41]$; $[M=8,0-20 \mathrm{~dB}, J=41] ;[M=16,0-25 \mathrm{~dB}, J=51] ;[M=32$, $0-30 \mathrm{~dB}, J=61] ;[M=64,0-30 \mathrm{~dB}, J=61]$

$M$-DPSK: [ $M=4,0-18 \mathrm{~dB}, J=37]$; $[M=8,0-24 \mathrm{~dB}, J=48]$; $[M=16,0-30 \mathrm{~dB}, J=61]$; $[M=32$, $0-30 \mathrm{~dB}, J=61]$

The number of sample measurements $J$ (for curve fitting) and the range of SNR should not be chosen to be too large as a wider range may lead to higher accuracy at the higher SNR values but with an unacceptable level of error predictions at the lower SNR values. The values for $a$ and $b$ in our exponential-type CEP formula (in closed-form) may be optimized for other ranges of SNR as well in a straight-forward fashion, but we believe that the optimized coefficients summarized in Table 1 and Table 2 are adequate for our applications.

Table 1. Exact and approximate CEP for coherent modulations.

\begin{tabular}{|l|l|l|}
\hline Modulation & Exact CEP $P_{s}(\varepsilon \mid \gamma)$ & $\begin{array}{l}\text { Approx. } \\
\text { CEP } a e^{-b \gamma}\end{array}$ \\
\hline M-PSK & $\frac{1}{\pi} \int_{0}^{\left(\frac{M-1) \pi}{M} \exp \left(\frac{-\gamma \sin ^{2}(\pi / M)}{\sin ^{2} \theta}\right) d \theta\right.}$ & \\
\hline BPSK & $Q(\sqrt{2 \gamma})$ & $\begin{array}{l}a=0.1669, \\
b=1.0493\end{array}$ \\
\hline QPSK & $2 Q(\sqrt{\gamma})-Q^{2}(\sqrt{\gamma})$ & $\begin{array}{l}a=0.4113, \\
b=0.5423\end{array}$ \\
\hline 8-PSK & $M=8$ & $\begin{array}{l}a=0.4682, \\
b=0.153\end{array}$ \\
\hline 16-PSK & $M=16$ & $\begin{array}{l}a=0.5008, \\
b=0.0405\end{array}$ \\
\hline 32-PSK & $M=32$ & $\begin{array}{l}a=0.6736, \\
b=0.0115\end{array}$ \\
\hline M-QAM & $4\left(\frac{\sqrt{M}-1}{\sqrt{M}}\right) Q\left(\sqrt{\frac{3 \gamma}{M-1}}\right)-4\left(\frac{\sqrt{M}-1}{\sqrt{M}}\right)^{2} Q^{2}\left(\sqrt{\frac{3 \gamma}{M-1}}\right)$ & \\
\hline 4-QAM & $2 Q(\sqrt{\gamma})-Q^{2}(\sqrt{\gamma})$ & $\begin{array}{l}a=0.4113, \\
b=0.5423\end{array}$ \\
\hline 8-QAM & $M=8$ & $\begin{array}{l}a=0.4552, \\
b=0.2305\end{array}$ \\
\hline 16-QAM & $M=16$ & $\begin{array}{l}a=0.6253, \\
b=0.1061\end{array}$ \\
\hline 32-QAM & $M=32$ & $\begin{array}{l}a=0.6678, \\
b=0.0516\end{array}$ \\
\hline 64-QAM & $M=64$ & $\begin{array}{l}a=0.7922, \\
b=0.0257\end{array}$ \\
\hline $\begin{array}{l}\text { Non-orthogonal } \\
\text { Binary FSK }\end{array}$ & $Q(\sqrt{1.217 \gamma})$ & $\begin{array}{l}a=0.1913, \\
b=0.6483\end{array}$ \\
\hline $\begin{array}{l}\text { Differentially } \\
\text { Encoded BPSK }\end{array}$ & $2 Q(\sqrt{2 \gamma})-2 Q^{2}(\sqrt{2 \gamma})$ & $\begin{array}{l}a=0.3335, \\
b=1.0492\end{array}$ \\
\hline $\begin{array}{l}\text { Differentially } \\
\text { Encoded QPSK }\end{array}$ & $4 Q(\sqrt{\gamma})-8 Q^{2}(\sqrt{\gamma})+8 Q^{3}(\sqrt{\gamma})-4 Q^{4}(\sqrt{\gamma})$ & $\begin{array}{l}a=0.6763, \\
b=0.5251\end{array}$ \\
\hline & & \\
\hline
\end{tabular}


International Journal of Wireless \& Mobile Networks (IJWMN) Vol. 4, No. 1, February 2012

Table 2. Exact and approximate CEP for non-coherent and differentially coherent modulations.

\begin{tabular}{|l|l|l|}
\hline Modulation & Exact CEP $P_{s}(\varepsilon \mid \gamma)$ & $\begin{array}{l}\text { Approx. } \\
\text { CEP } a e^{-b \gamma}\end{array}$ \\
\hline M-FSK & $\sum_{n=1}^{M-1}(-1)^{n+1}\left(\begin{array}{c}M-1 \\
n\end{array}\right) \frac{1}{n+1} \exp \left(-\frac{n}{n+1} \gamma\right)$ & \\
\hline M-DPSK & $\frac{1}{2 \pi} \int_{0}^{\left(\frac{M-1) \pi}{M}\right.} \exp \left(\frac{-\gamma \sin ^{2}(\pi / M)}{\sin ^{2} \theta+\sin ^{2}(\theta+\pi / M)}\right) d \theta$ & \\
\hline 2-DPSK & $0.5 e^{-\gamma}$ & $\begin{array}{l}a=0.5000, \\
b=1.0000\end{array}$ \\
\hline 4-DPSK & $M=4$ & $\begin{array}{l}a=0.2491, \\
b=0.3185\end{array}$ \\
\hline 8-DPSK & $M=8$ & $\begin{array}{l}a=0.5382, \\
b=0.0838\end{array}$ \\
\hline 16-DPSK & $M=16$ & $\begin{array}{l}a=0.5955, \\
b=0.0214\end{array}$ \\
\hline 32-DPSK & $M=32$ & $\begin{array}{l}a=0.7511, \\
b=0.0064\end{array}$ \\
\hline
\end{tabular}

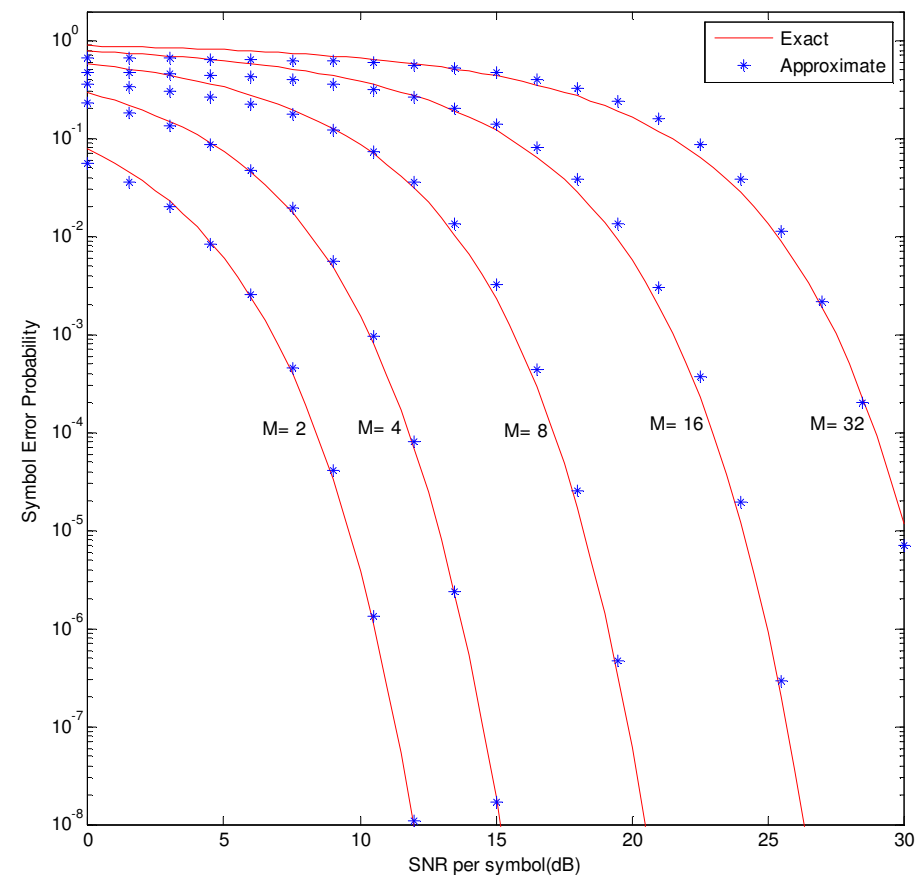

Fig. 1. Comparisons between the exact and approximate CEP expressions for coherent MPSK modulation $(M=2,4,8,16$ and 32) in an AWGN channel. 
International Journal of Wireless \& Mobile Networks (IJWMN) Vol. 4, No. 1, February 2012

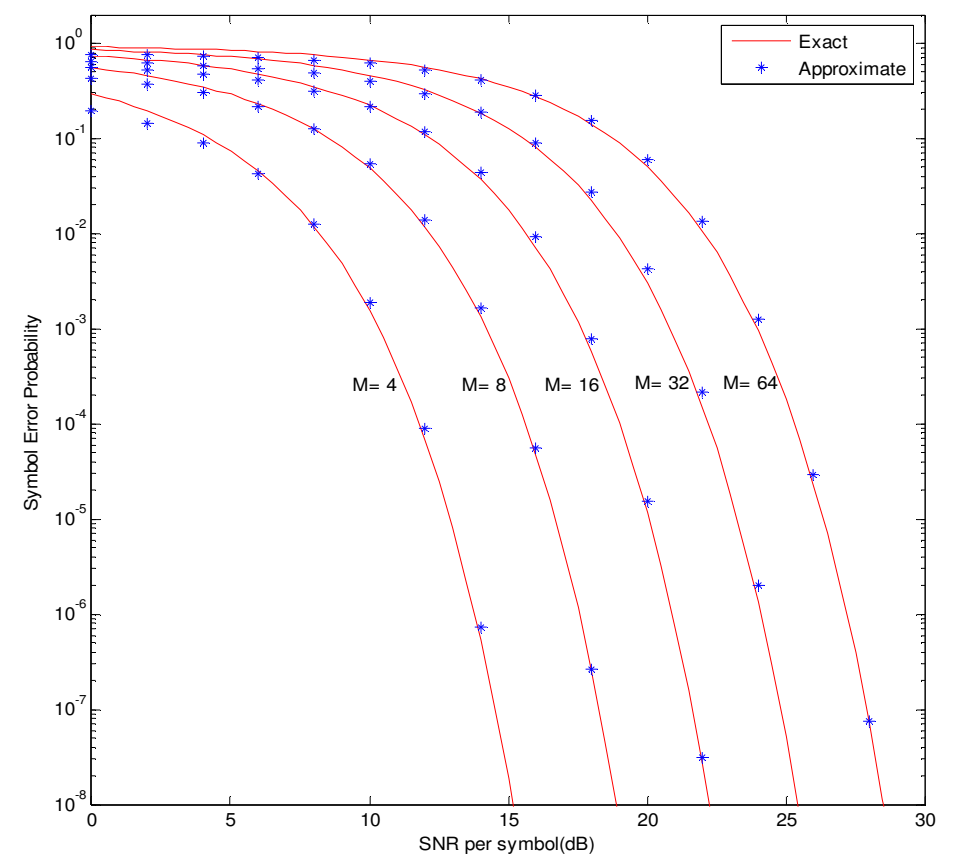

Fig. 2. Comparisons between the exact and approximate CEP expressions for coherent MQAM modulation $(M=4,8,16,32$ and 64) in an AWGN channel.

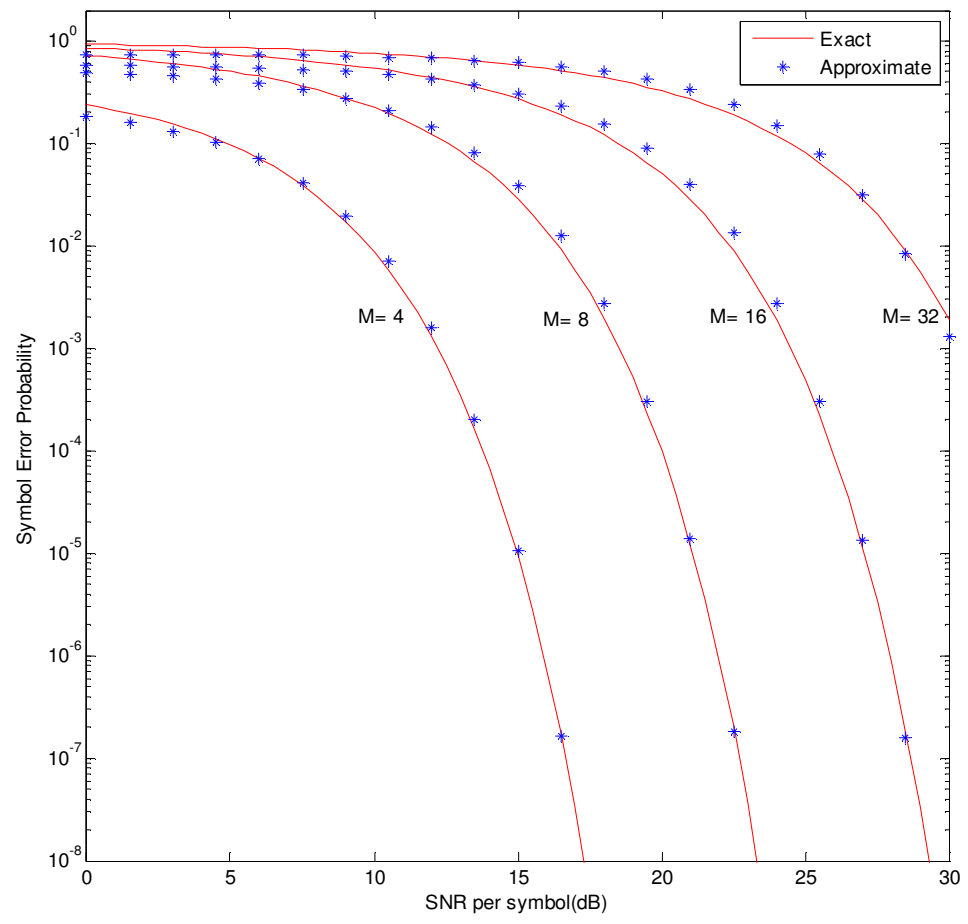

Fig. 3. Comparisons between the exact and approximate CEP expressions for differentially coherent MPSK modulation $(M=4,8,16$, and 32) in an AWGN channel. 
International Journal of Wireless \& Mobile Networks (IJWMN) Vol. 4, No. 1, February 2012

\section{ASYMPTOTIC ASER ANALYSIS FOR CAF RELAY SySTEM}

This section details our approach for deriving a tight asymptotic ASER of 2-hop CAF multirelay networks over generalized fading channels. The non-cooperative diversity case is first studied and subsequently, our analysis is extended to 2-hop multi-relay CAF networks.

\subsection{No Relay (Source to Destination Link) Case}

Using our exponential-type CEP approximation in Table 1 and Table 2, it is straight forward to show that the ASER is given by

$$
P_{s}(\varepsilon) \approx a \phi_{\gamma}(b ; \Omega)
$$

since the resulting averaging expression is simply the Laplace transform of the PDF of SNR.

Example 1: For the sake of clarity on the efficacy of approach even for the non-cooperative diversity case, next we will illustrate various approximations and asymptotic formula for BPSK modulation over a Nakagami-m channel. Using our CEP approximation approach, we obtain

$$
P_{S}(\varepsilon) \approx a(1+b \Omega / m)^{-m}
$$

where $a=0.1669$ and $b=1.0493$ for BPSK from Table 1 , and $m$ denotes the fading severity parameter. Similarly, an asymptotic approximate ASER is given by replacing the MGF with its corresponding asymptotic MGF. The exact and asymptotic MGF for all common fading models are listed in Table 3.

The corresponding exact ASER of BPSK in Nakagami-m channel is given by [13]

$$
P_{s}(\varepsilon)=\frac{1}{\pi} \int_{0}^{\pi / 2}\left(1+\frac{\Omega}{m \sin ^{2} \theta}\right)^{-m} d \theta
$$

Moreover, using the asymptotic analysis technique proposed in [6], we obtain

$$
P_{S}(\varepsilon) \approx \frac{m^{m-1} \Gamma(m+1 / 2)}{2 \sqrt{\pi} \Gamma(m)} \Omega^{-m}
$$

Our approximation for the ASER (4), the exact ASER formula (5), and the asymptotic ASER (6) are compared in Fig. 4 for several values of the fading severity index (i.e., $m=0.5,1,2$ and 4). Although the asymptotic approximation (6) performs better than (4) in severely faded environments, we observe that our approach is very good at moderate and large values of $m$. The gap at small $m$ values is mainly attributed to the CEP approximation in closed-form since the MGF of SNR is exact. Thus we anticipate that a tighter ASER approximation may be derived if one or additional exponential terms are considered to approximate the CEP in (2). It is also apparent from Fig. 4 that unlike the asymptotic ASER (6) case, the curves corresponding to the approximate ASER technique (i.e., Eq. (4)) follows the trend of the exact ASER case for entire range of mean received SNR/symbol. Hence, this particular form is particularly attractive for evaluating the ASER outage metric (which is defined as the probability that the actual ASER does not meet its target ASER value) in a shadowed fading environment since (4) is invertible with respect to its mean received SNR $\Omega$. Although not shown in Fig. 4, we also observed that the curves from the approximate ASER given by (3) in conjunction with the asymptotic MGF on Table 3 yields comparable performance with that of (6) especially at higher values of $m$. Thus, our approach to the derivation of an accurate unified ASER expression (in closed-form) also naturally leads to yet an alternative asymptotic ASER formula for different digital modulations over generalized fading channels. 
International Journal of Wireless \& Mobile Networks (IJWMN) Vol. 4, No. 1, February 2012

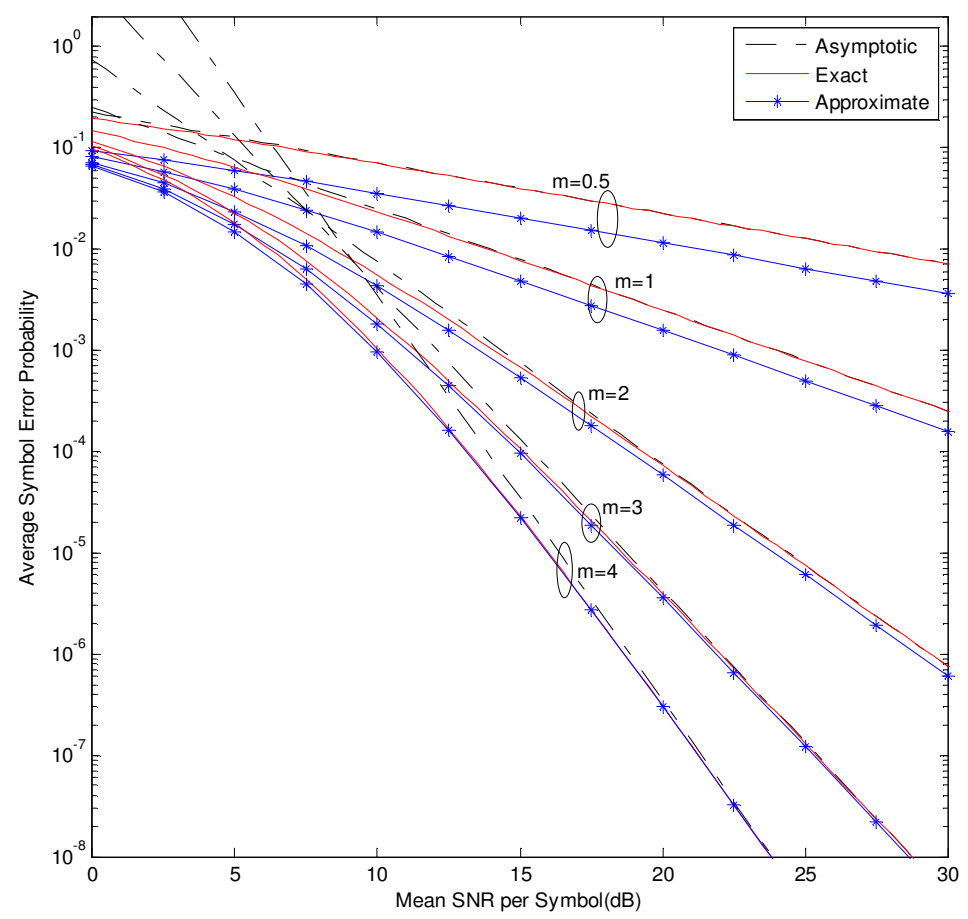

Fig. 4. Comparison of asymptotic, approximate and exact ASER of non-cooperative diversity system with BPSK $(a=0.1669, b=1.0493)$ over Nakagami-m channels.

Table 3. Exact and asymptotic expressions for the MGF and PDF of SNR.

\begin{tabular}{|l|l|l|l|}
\hline Fading Type & $\begin{array}{l}\text { Exact MGF } \\
\phi_{\gamma}(s)=\int_{0}^{\infty} f(\gamma) e^{-s \gamma} d \gamma\end{array}$ & $\begin{array}{l}\text { Asymptotic MGF } \\
\tilde{\phi}_{\gamma}(s)=t_{1} \Gamma\left(t_{2}+1\right) s^{-\left(t_{2}+1\right)}\end{array}$ & $\begin{array}{l}\text { Asymptotic PDF } \\
f(\gamma)=t_{1} \gamma^{2}\end{array}$ \\
\hline Rayleigh & $(1+s \Omega)^{-1}$ & $t_{2}=0, t_{1}=1 / \Omega$ & $t_{2}=0, t_{1}=1 / \Omega$ \\
\hline $\begin{array}{l}\text { Nakagami- } q \\
\text { (Hoyt) }\end{array}$ & $\left(1+2 s \Omega+\frac{4 s^{2} \Omega^{2} q^{2}}{\left(1+q^{2}\right)^{2}}\right)^{-1 / 2}$ & $t_{2}=0, t_{1}=\frac{1+q^{2}}{2 q \Omega}$ & $t_{2}=0, t_{1}=\frac{1+q^{2}}{2 q \Omega}$ \\
\hline $\begin{array}{l}\text { Nakagami- } n \\
\left.\text { (Rice } K=n^{2}\right)\end{array}$ & $\frac{1+K}{1+K+s \Omega} \exp \left(\frac{-K s \Omega}{1+K+s \Omega}\right)$ & $t_{2}=0, t_{1}=\frac{(1+K) e^{-K}}{\Omega}$ & $t_{2}=0, t_{1}=\frac{(1+K) e^{-K}}{\Omega}$ \\
\hline Nakagami- $m$ & $(1+s \Omega / m)^{-m}$ & $t_{2}=m-1, t_{1}=\frac{m^{m}}{\Gamma(m) \Omega^{m}}$ & $t_{2}=m-1, t_{1}=\frac{m^{m}}{\Gamma(m) \Omega^{m}}$ \\
\hline
\end{tabular}

\subsection{Two-Hops Cooperative Amplify-and-Forward Multi-Relay Networks}

It has been shown in [8] that the total end-to-end received SNR of a two-hop CAF diversity system with $N$ relays (assuming a maximal-ratio combining receiver) is given by

$$
\gamma_{T}=\gamma_{s, d}+\sum_{i=1}^{N} \frac{\gamma_{s, i} \gamma_{i, d}}{1+\gamma_{s, i}+\gamma_{i, d}} \cong \gamma_{s, d}+\sum_{i=1}^{N} \tilde{\gamma}_{k}
$$


where $\tilde{\gamma}_{k}=\frac{\gamma_{s, k} \gamma_{k, d}}{\gamma_{s, k}+\gamma_{k, d}}$ and, $\gamma_{s, d}, \gamma_{s, i}, \gamma_{i, d}$ denote the instantaneous received SNR for the source-destination link, source-relay link and relay-destination link, respectively. However, it has been recognized in the literature that the evaluation of the PDF of harmonic mean SNR (7) is very challenging and the exact ${ }^{1}$ PDF of CAF relayed path is only available for either the Rayleigh [1]-[3] or Nakagami-m channels with i.i.d fading statistics [4]. Bounds such as $\frac{1}{2} \min \left(\gamma_{s, k}, \gamma_{k, d}\right) \leq \gamma_{k} \leq \min \left(\gamma_{s, k}, \gamma_{k, d}\right)$ have also been developed to overcome the analytical difficulty of finding the desired PDF with independent but non-identically distributed (i.n.d) wireless links in Nakagami-m [9][10] and Rice [11] channels or to simplify analysis in Rayleigh fading [2][8].

For the sake of illustration, let us first consider a CAF network with a single relay $(N=1)$. In this case, the effective end-to-end SNR is given by

$$
\gamma_{T}=\gamma_{s, d}+\frac{\gamma_{s, 1} \gamma_{1, d}}{1+\gamma_{s, 1}+\gamma_{1, d}}=\gamma_{s, d}+\gamma_{1}
$$

The cumulative distribution function (CDF) of $\gamma_{1}$ has been shown in [10] to be upper-bounded by the CDF of $\min \left(\gamma_{s, k}, \gamma_{k, d}\right)$ given by

$$
F_{\gamma_{1}}(\gamma) \leq F_{\gamma_{s, 1}}(\gamma)+F_{\gamma_{1, d}}(\gamma)-F_{\gamma_{s, 1}}(\gamma) F_{\gamma_{1, d}}(\gamma)
$$

Since the values of $F_{\gamma_{s, 1}}(\gamma)$ and $F_{\gamma_{1, d}}(\gamma)$ range between 0 and 1, the product of these two CDFs will be much less than their addition. Hence, we can derive an approximate $\mathrm{MGF}^{2}$ of $\gamma_{1}$ by neglecting the product term in (9), viz.,

$$
\phi_{\gamma_{1}}(s) \approx \phi_{\gamma_{s, 1}}(s)+\phi_{\gamma_{1, d}}(s)
$$

Then using the exponential approximation of CEP in (2), it is straight-forward to show that the end-to-end ASER of a two-hops CAF relay system with direct source-to-destination transmission can be approximated as

$$
P_{s}(\varepsilon) \approx a \phi_{\gamma_{s, d}}\left(b ; \Omega_{s d}\right)\left(\phi_{\gamma_{s, 1}}\left(b ; \Omega_{s, 1}\right)+\phi_{\gamma_{1, d}}\left(b ; \Omega_{1, d}\right)\right)
$$

For the special case of i.i.d. paths, (11) reduces into

$$
P_{s}(\varepsilon) \approx 2 a \phi_{\gamma}^{2}(b ; \Omega)
$$

It is rather straight-forward to extend our asymptotic analysis for $N$ cooperating relays. It this case, the ASER is given by

$$
P_{s}(\varepsilon) \approx a \phi_{\gamma_{s, d}}\left(b ; \Omega_{s, d}\right) \prod_{i=1}^{N}\left(\phi_{\gamma_{s, i}}\left(b ; \Omega_{s, i}\right)+\phi_{\gamma_{i, d}}\left(b ; \Omega_{i, d}\right)\right)
$$

and for the i.i.d. case, we obtain

\footnotetext{
1 To the best of our knowledge, all previous work for the 'exact' PDF of the relayed path is computed as $\tilde{\gamma}_{k}=\gamma_{s, k} \gamma_{k, d} /\left(\gamma_{s, k}+\gamma_{k, d}\right)$, which becomes very accurate for large SNR.

${ }^{2}$ It is pointed out that a slightly tighter approximation for the MGF $\phi_{\gamma_{1}}(s)$ can be obtained (from (9)) as $\phi_{\gamma_{1}}(s) \approx \phi_{\gamma_{s, 1}}(s)+\phi_{\gamma_{1, d}}(s)-\phi_{\gamma_{s, 1}}(s) \phi_{\gamma_{1, d}}(s)$. The investigation on the efficacy of this alternative approximation is beyond the scope of this article, but it will be pursued as part of our future work.
} 
International Journal of Wireless \& Mobile Networks (IJWMN) Vol. 4, No. 1, February 2012

$$
P_{s}(\varepsilon) \approx 2^{N} a\left(\phi_{\gamma_{s, d}}\left(b ; \Omega_{s, d}\right)\right)^{N+1}
$$

In the following, we will discuss the efficacy of our asymptotic approximation technique for the ASER of CAF networks by comparing it with other known approximations, asymptotic formulas and bounding methods for coherent BPSK modulation scheme (only chosen for illustrative purpose) over Rayleigh, Nakagami- $m$ and Rician channels.

The exact ASER is given by [2, eq. (6)]

$$
P_{s}(\varepsilon)=\frac{1}{\pi} \int_{0}^{(M-1) \pi / M} \phi_{\gamma_{s, d}}\left(\frac{g_{P S K}}{\sin ^{2} \theta} ; \Omega_{s, d}\right) \prod_{i=1}^{N}\left(\phi_{\tilde{\gamma}_{i}}\left(\frac{g_{P S K}}{\sin ^{2} \theta} ; \Omega_{i}\right)\right) d \theta
$$

where $g_{P S K}=\sin ^{2}(\pi / M)$ and $M$ denotes the constellation size. The "exact" MGF $\phi_{\bar{\gamma}_{i}}($.$) is only$ known for several special cases as discussed in Sections 1 and 2.

It is also important to note that the asymptotic formula, [5, eq. (32)] (hereafter, referred to as RCG method) is not applicable for the Nakagami- $m$ channel since $t_{2}=m-1$ and thus $f_{\gamma}(\gamma ; \Omega, h)=t_{1} \gamma^{t_{2}}=0$ when $\gamma=0$. Consequently, the expression shown in (16),

$$
P_{s}(\varepsilon) \approx O(N) f_{\gamma_{s, d}}(0) \prod_{i=1}^{N}\left(f_{\gamma_{s, 1}}(0)+f_{\gamma_{1, d}}(0)\right)
$$

where $O(N)=\left(\prod_{n=1}^{N+1}(2 n-1)\right) /\left(2(N+1) ! 2^{N+1}\right)$, cannot be used for analysis over Nakagami- $m$ channel except for $m=1$. This problem, however, has been addressed in [14]. To obtain a more general solution, we develop an alternative asymptotic formula similar to [5] for generalized fading channels using our approximation in (10), viz.,

$$
P_{s}(\varepsilon) \approx \frac{1}{\pi} \int_{0}^{\pi / 2} \tilde{\phi}_{\gamma_{s, d}}\left(\frac{1}{\sin ^{2} \theta} ; \Omega_{s, d}\right) \prod_{i=1}^{N}\left[\tilde{\phi}_{\gamma s, i}\left(\frac{1}{\sin ^{2} \theta} ; \Omega_{s, i}\right)+\tilde{\phi}_{\gamma i, d}\left(\frac{1}{\sin ^{2} \theta} ; \Omega_{i, d}\right)\right] d \theta
$$

However, for the special case of i.n.d fading statistics where all channels has same fading index but different average SNRs, the above integral can be further simplified into a closed-form expression, viz.,

$$
P_{s}(\varepsilon) \approx O\left(\left(t_{2}+1\right)(N+1)-1\right) t_{3}^{N+1} \frac{1}{\Omega_{s, d}^{t_{2}+1}} \prod_{i=1}^{N}\left(\frac{1}{\Omega_{s, i}^{t_{2}+1}}+\frac{1}{\Omega_{i, d}^{t_{2}+1}}\right)
$$

where $O(N)$ is defined in [5, eq. (32)], $t_{3}=t_{1} \Gamma\left(t_{2}+1\right) \Omega^{t_{2}+1}$ and $t_{1}, t_{2}$ for several fading channel models are summarized in Table 3. Interestingly, (18) reduces to the same expression as in [5, eq. (33)] (after correcting ${ }^{3}$ a typo) for the i.n.d Rice case. Thus, our asymptotic formula (18) can be viewed as a generalization of [5] since it also encapsulates the Nakagami-m case (hereafter, referred to as 'modified RCG' method).

Example 2: (Nakagami-m channel) Consider a CAF network with BPSK modulation in a Nakagami-m fading environment. The exact ASER expression for i.i.d. Nakagami- $m$ channel can be shown as [4, eq. (26)]

$$
P_{s}(\varepsilon)=\frac{1}{\pi} \int_{0}^{\pi / 2}\left(1+\frac{\Omega_{s, d}}{m \sin ^{2} \theta}\right)^{-m}\left({ }_{2} F_{1}\left(m, 2 m ; m+\frac{1}{2} ; \frac{-\Omega_{1}}{4 m \sin ^{2} \theta}\right)\right)^{N} d \theta
$$

\footnotetext{
${ }^{3}$ In [5, eq.(33)], an exponential term $e^{-(N+1) K}$ was missing and this explains why only the Rayleigh fading case $(K=0)$ was considered for numerical computations because only this case can be evaluated correctly.
} 
International Journal of Wireless \& Mobile Networks (IJWMN) Vol. 4, No. 1, February 2012

where ${ }_{2} F_{1}(., ., . ;$.$) is the Gauss Hypergeometric function [35]. Also the "exact" MGF \phi_{\gamma_{i}}(s)$ in Rayleigh ( $m=1)$ fading with i.n.d fading statistics is given by [3, eq. (52)]

$\phi_{\gamma_{i}}(s)=\left[\left(1 / \Omega_{s, i}-1 / \Omega_{i, d}\right)^{2}+\left(1 / \Omega_{s, i}-1 / \Omega_{i, d}\right) s\right] / \Delta^{2}+\frac{2 s}{\Delta^{3} \Omega_{s, i} \Omega_{i, d}} \ln \left(\left(s+\Delta+\frac{1}{\Omega_{s, i}}+\frac{1}{\Omega_{i, d}}\right)^{2} \frac{\Omega_{s, i} \Omega_{i, d}}{4}\right)$

where $\Delta=\sqrt{\left(1 / \Omega_{s, i}-1 / \Omega_{i, d}\right)^{2}+2 s\left(1 / \Omega_{s, i}+1 / \Omega_{i, d}\right)+s^{2}}$.

Moreover, the exact MGF $\phi_{\gamma_{i}}(s)$ for Nakagami-m with i.n.d fading statistics can be evaluated numerically using the results in [15, Eq. (5) and Table 1]. Bounding technique based on upper and lower bounds of the effective SNR [8]-[11] will also be presented to validate the accuracy of our approach. The MGF for upper bound of the effective SNR of two-hop relayed path can be expressed as [10, eq. (11)]

$$
\begin{gathered}
\phi_{\tilde{\gamma}_{i}}(s)=\sum_{\substack{\mathrm{k} \in\{(\mathrm{s}, 1),(1, \mathrm{~d})\} \\
j \neq k}} \frac{\Gamma\left(m_{k}+m_{j}\right)}{\Gamma\left(m_{k}\right) \Gamma\left(m_{j}\right)}{ }_{2} F_{1}\left(1-m_{j}, m_{k} ; 1+m_{k} ; \frac{\left(s \Omega_{k}+m_{k}\right) \Omega_{j}}{s \Omega_{j} \Omega_{k}+\Omega_{j} m_{k}+\Omega_{k} m_{j}}\right) \\
\times \frac{1}{m_{k}}\left(\frac{\Omega_{j} m_{k}}{s \Omega_{j} \Omega_{k}+\Omega_{j} m_{k}+\Omega_{k} m_{j}}\right)^{m_{k}}
\end{gathered}
$$

and the lower bound is given by $\phi_{\hat{\gamma}_{i}}(s / 2)$. For this case, the ASER will be evaluated as (i.e., to mask out the effect of CEP approximation in (13))

$$
P_{s}(\varepsilon) \approx \frac{1}{\pi} \int_{0}^{\pi / 2} \phi_{\gamma_{s, d}}\left(\frac{1}{\sin ^{2} \theta} ; \Omega_{s, d}\right) \prod_{i=1}^{N} \phi_{\gamma_{i}}\left(\frac{1}{\sin ^{2} \theta} ; \Omega_{i}\right) d \theta
$$

Fig. 5 and Fig. 6 compare the curves corresponding to bounding and asymptotic analyses methods discussed in the above example over Nakagami-m channels ( $m=1$ and 3$)$ with either a single relay with i.i.d fading statistics or two relays with independent but non-identically distributed (i.n.d) fading statistics, respectively.

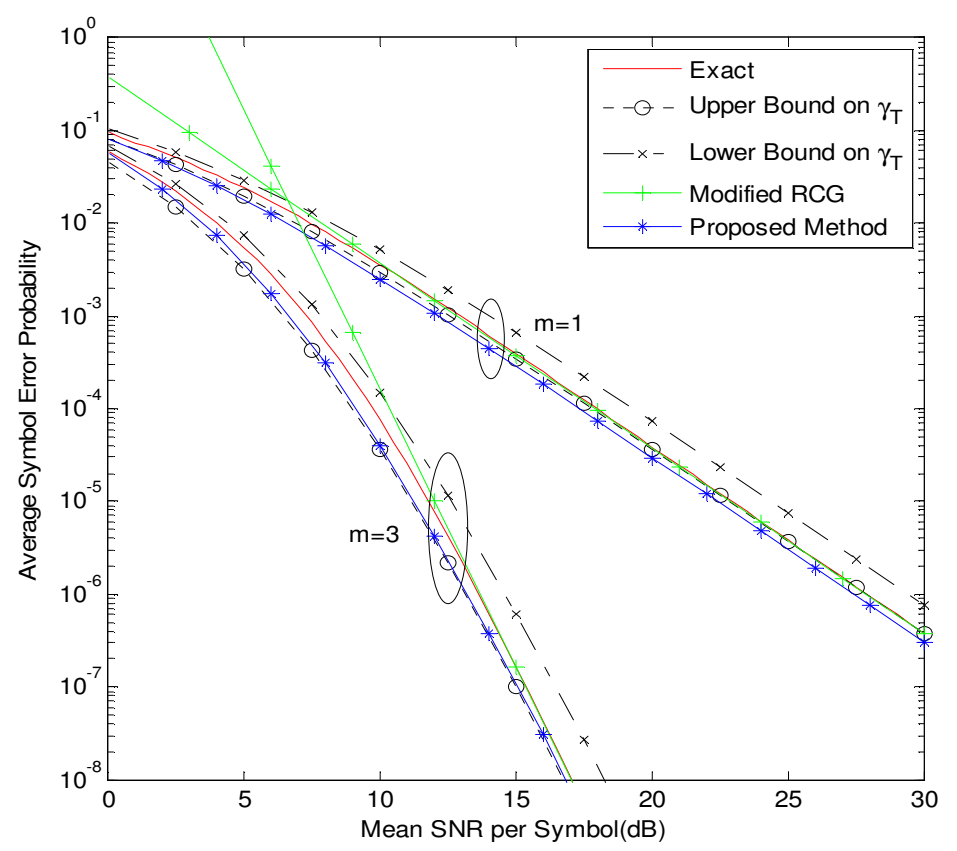

Fig. 5. ASER of BPSK signal over i.i.d. Nakagami channel ( $m=1$ and 3) with a single cooperative amplify-and-forward relay. 


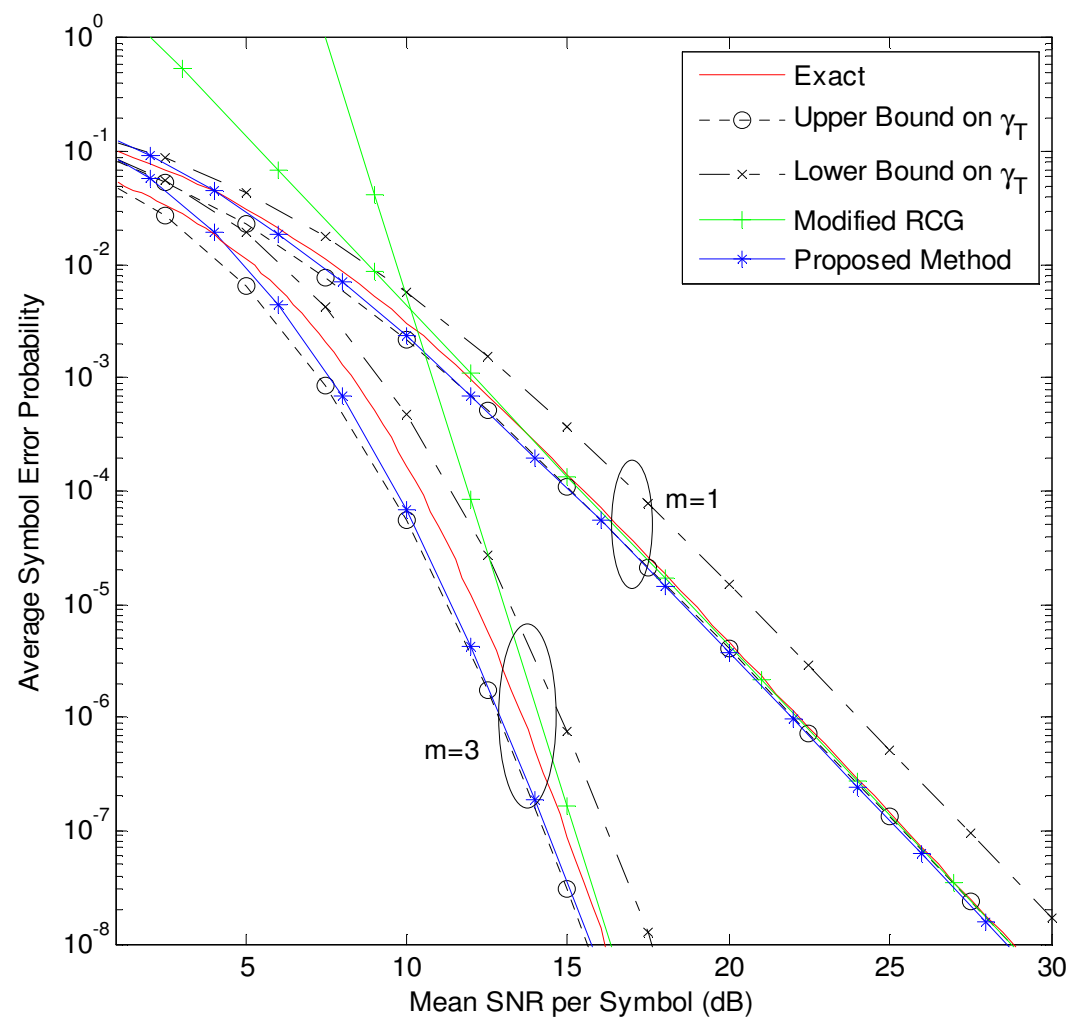

Fig. 6. ASER of BPSK signal over i.n.d Nakagami-m $(m=1$ and 3$)$ fading environments with two CAF relays and the mean link SNRs are given by $\Omega_{s, d}=0.4 \Omega_{T}, \Omega_{s, 1}=\Omega_{T}, \Omega_{s, 2}=\Omega_{T}$, $\Omega_{1, d}=0.43 \Omega_{T}$ and $\Omega_{2, d}=0.43 \Omega_{T}$.

While our approximation (13) is much closer to the "upper bound" case (as evident from Fig. 5 and Fig. 6), it performs considerably better than any other approximations or asymptotic analysis especially at low mean SNRs and at higher values of the fading severity index. This observation is very interesting given that cooperative transmission schemes are typically employed to decrease/conserve the transmit power at the source, and thus the performance in the low SNR regime is of particular interest. Besides, our ASER formula (13) is in closed-form, and can handle many fading environments that heretofore resisted a simple solution (e.g., mixed-fading environments, composite-fading, etc.)!

Example 3: (Rice channel) Consider a CAF network with BPSK modulation in a Rice (Nakagami-n) fading environment. In this case, the "exact" expression for the MGF of SNR for the relayed path presented in [15, Eq. (5) and Table 1] does not appear to be numerically stable or efficient (due to the need to perform numerical integration of product of two infinite series). Thus, the bounding technique [11] and asymptotic analysis [5] appear to be the only known viable analytical tools for the ASER analysis. In order to provide a comprehensive performance comparison, we also generated ASER curves corresponding to the Monte Carlo simulations in Rice channels. The MGF of SNR (upper bound) for the relayed path with two-hops has been derived in [11] and is given by

$$
\phi_{\tilde{\gamma}_{i}}(s)=\sum_{\substack{k \in\{(s, i)(i, d)\} \\ j \neq k}} 2 A_{k} e^{-K_{k}} I\left(\sqrt{2 A_{j}}, \sqrt{2 K_{j}}, \sqrt{2 A_{k} K_{k}}, 2\left(s+A_{k}\right)\right)
$$

where $A_{i}=\left(1+K_{i}\right) / \Omega_{i}$, and 
International Journal of Wireless \& Mobile Networks (IJWMN) Vol. 4, No. 1, February 2012

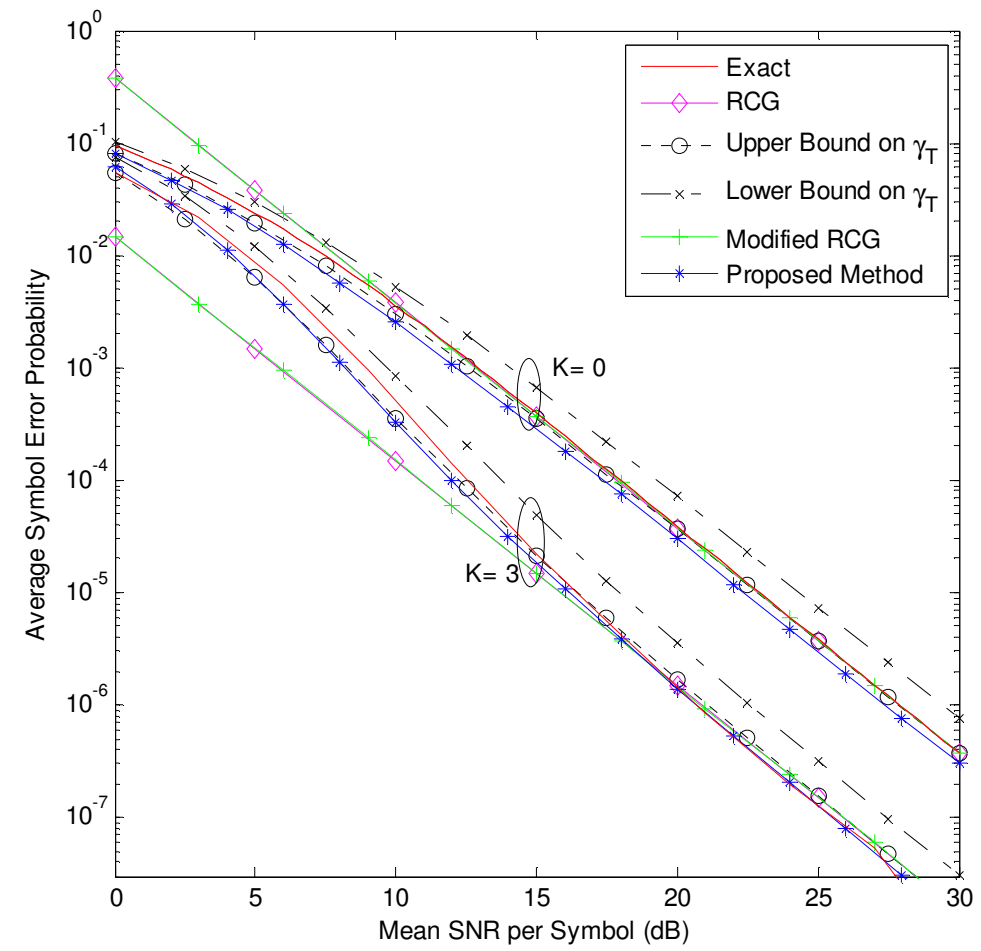

Fig. 7. ASER of BPSK signal over i.i.d. Rician channels $(K=0$ and $K=3)$ for a single cooperative relay.

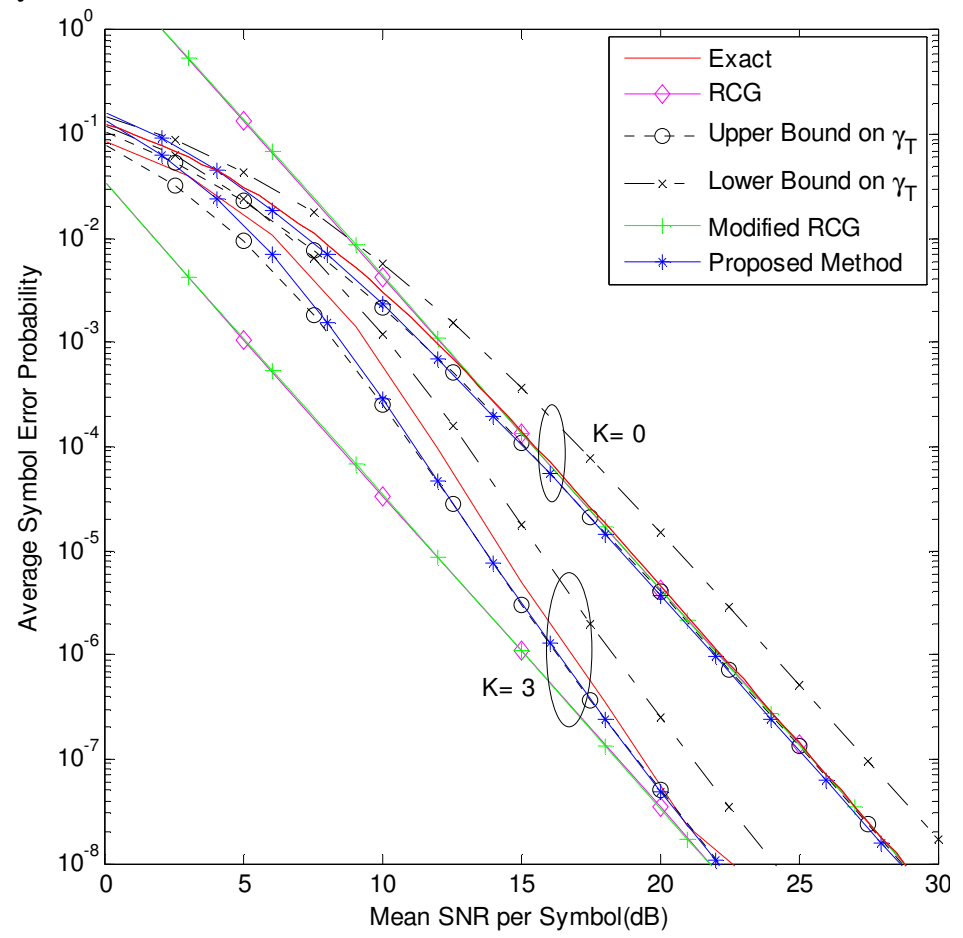

Fig. 8. ASER of CAF with BPSK over i.n.d Rice channels $(K=0$ and $K=3)$ for two cooperative relays (mean link SNRs are assumed to be $\Omega_{s, d}=0.4 \Omega_{T}, \Omega_{s, 1}=\Omega_{T}, \Omega_{s, 2}=\Omega_{T}$, $\Omega_{1, d}=0.43 \Omega_{T}$ and $\left.\Omega_{2, d}=0.43 \Omega_{T}\right)$. 
International Journal of Wireless \& Mobile Networks (IJWMN) Vol. 4, No. 1, February 2012

$$
I[a, b, c, d]=\frac{1}{d} e^{\frac{c^{2}}{2 d}} Q\left(b \sqrt{\frac{d}{\left(d+a^{2}\right)}}, \frac{a c}{\sqrt{d\left(d+a^{2}\right)}}\right)-\frac{a^{2}}{d\left(d+a^{2}\right)} e^{\frac{c^{2}-b^{2} d}{2\left(d+a^{2}\right)}} I_{0}\left(\frac{a b c}{\left(d+a^{2}\right)}\right),
$$

where $Q(.,$.$) is first order Marcum Q-function and I_{0}($.$) is the modified Bessel function of the$ first kind. The MGF for the lower bound case may be computed as $\phi_{\hat{\gamma}_{1}}(s / 2)$ while the curves corresponding to the asymptotic analyses approaches will be generated using (18) (i.e., modified RCG method) or using (13) (i.e., our approach).

Fig. 7 and Fig. 8 compare various approximations and bounds for the ASER of CAF in both Rayleigh $(K=0)$ and Rice channels $(K=3)$. The utility of our approximation (13) in this problem is evident, especially at the lower mean SNR values. Thus, one may confidently utilize (13) for system-level optimization of cooperative relay networks (i.e., owing to its simplicity as well as for predicting ASER with a good accuracy) to address important issues such as joint optimization of power allocation and relay placement in wireless sensor networks and/or cellular network with sparsely distributed base-stations.

Although the results in Fig. 4 through Fig. 8 were generated for BPSK modulation, the trend as well as the complexity for evaluating the desired ASER for higher order modulations using (13) is expected to be the same as that for BPSK (because we only need to choose an appropriate values for $a$ and $b$ from Table 1 and Table 2). As an example, Fig. 9 depicts the ASER performance of CAF that employ a single cooperating relay in a Nakagami-m environment with i.i.d fading statistics. The trends (in terms of the tightness of the curves) are similar to that of observed in Fig. 5, as expected.

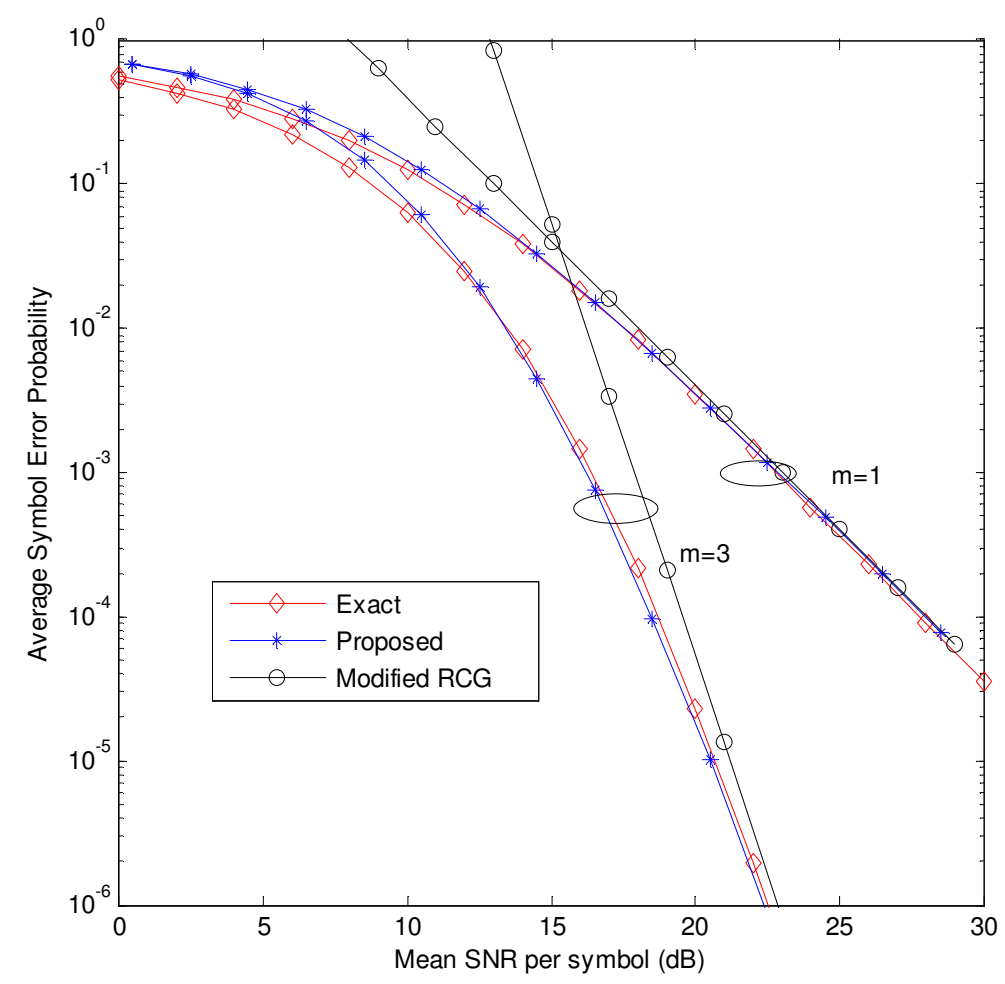

Fig. 9. ASER of CAF relay networks over i.i.d Nakagami-m fading environments with $N=1$ and 8-PSK modulation. 
International Journal of Wireless \& Mobile Networks (IJWMN) Vol. 4, No. 1, February 2012

\section{Symbol Error Outage Probability}

The symbol error outage $P_{\text {out }}$ is defined as the probability that the instantaneous error rate will exceed a specified target value $P_{e}^{*}$ [36]. This is equivalent to the probability that the instantaneous end-to-end received signal SNR at the destination node falls below a predetermined threshold $\gamma^{*}$ (which is specific to the chosen modulation), viz.,

$$
P_{\text {out }}=\int_{0}^{\gamma^{*}} f_{\gamma}(\gamma) d \gamma=F_{\gamma}\left(\gamma^{*}\right)
$$

Since the evaluation of the PDF of end-to-end SNR in CAF relay networks with maximal-ratio combining diversity receiver is generally cumbersome, it is much more convenient to the evaluate its CDF from the MGF using a robust Laplace transform inversion technique such as the fixed-Talbot method [38] as

$$
P_{\text {out }}=F_{\gamma}\left(\gamma^{*}\right)=\int_{0}^{\gamma^{*}} \mathfrak{I}^{-1}\left[\phi_{\gamma_{T}}(s)\right] d \gamma=\left.\mathfrak{I}^{-1}\left[\phi_{\gamma_{T}}(s) / s\right]\right|_{\gamma^{*}}
$$

where $\mathfrak{I}^{-1}$ (.) denotes the inverse Laplace transform. Readers are referred to [38] for additional details on the multi-precision Laplace inversion method.

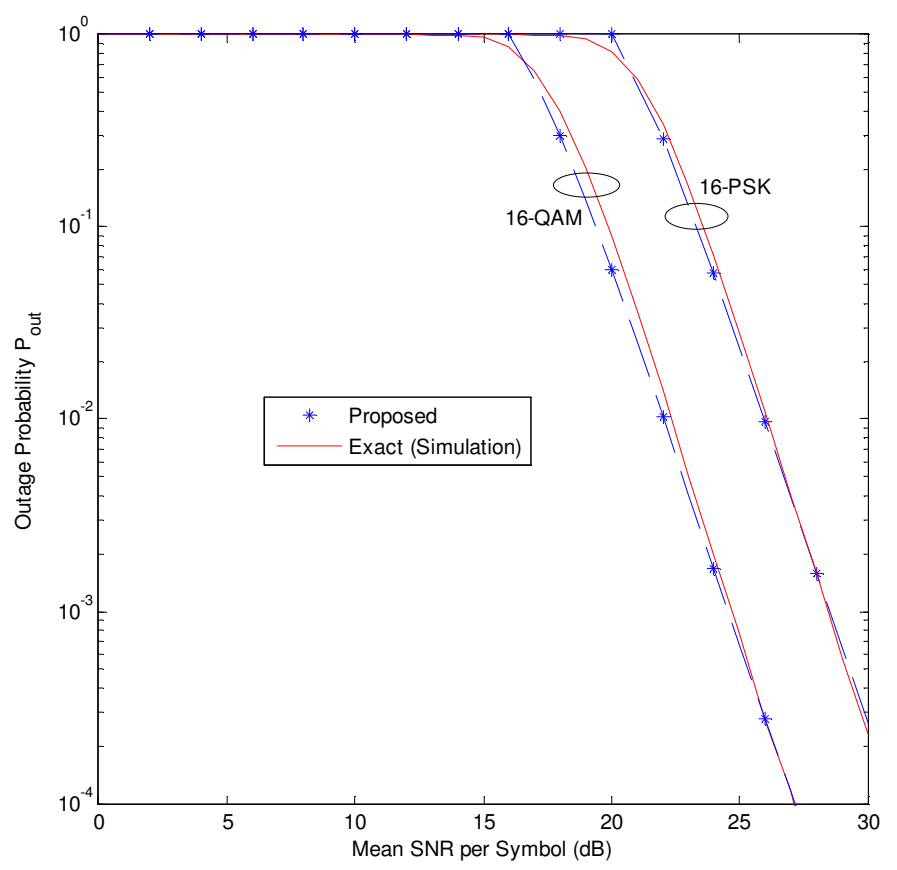

Fig. 10. Symbol error outage (for target symbol error rate of 0.01 ) for 16-QAM and 16-PSK over i.n.d Rice channels (Rice factor $K=3$ ) with two cooperative relays (mean link SNRs are assumed to be $\Omega_{s, d}=0.4 \Omega_{T}, \Omega_{s, 1}=\Omega_{T}, \Omega_{s, 2}=\Omega_{T}, \Omega_{1, d}=0.43 \Omega_{T}$ and $\Omega_{2, d}=0.43 \Omega_{T}$ ).

Fig. 10 shows a comparison between the exact and approximate analyses for the computation of symbol error outage with two CAF relays that employ either 16-PSK or 16-QAM in an i.n.d Rice fading environment, and the target SER is $10^{-2}$. For the approximate analysis, we compute the threshold in (25) for a broad class of digital modulations schemes as

$$
\gamma^{*}=\frac{1}{b} \ln \left(a / P_{e}^{*}\right)
$$


International Journal of Wireless \& Mobile Networks (IJWMN) Vol. 4, No. 1, February 2012

where the coefficients $a$ and $b$ for a number of digital modulation schemes are listed in Table 1 and Table 2. For instance, $(a=0.6253, b=0.1061)$ and $(a=0.5008, b=0.0405)$ for 16-QAM and 16-PSK respectively, which are used to generate the corresponding curves in Fig. 10. To generate the curves corresponding to the exact analysis, we employ Monte Carlo simulation technique with $10^{6}$ samples since an exact MGF of end-to-end SNR in Rice fading is not available. In this case, the threshold $\gamma^{*}$ for M-PSK and M-QAM modulation schemes are obtained via (27) and (28), respectively [38, Table II]:

$$
\begin{aligned}
& \gamma^{*}=\frac{1}{2}\left[\frac{Q^{-1}\left(P_{e}^{*} / 2\right)}{\sin (\pi / M)}\right]^{2} \\
& \gamma^{*}=\frac{(M-1)}{3}\left\{Q^{-1}\left[\frac{\sqrt{M}}{\sqrt{M}-1}\left(\frac{1-\sqrt{\left(1-P_{e}^{*}\right)}}{2}\right)\right]\right\}^{2}
\end{aligned}
$$

where $Q^{-1}$ (.) denotes the inverse of Gaussian Q-function. It is evident from Fig. 10 that even with two approximations (one from the CEP and the other from the MGF of SNR relayed path), our analytical approach still closely approximates the exact symbol error outage performance curves obtained via Monte Carlo simulation. Fig. 10 also shows that constant-envelope 16-PSK modulation experience a greater symbol error outage compared to the 16-QAM for the same target error rate (i.e., $P_{e}^{*}=0.01$ ) and mean received SNR/symbol, as anticipated. At higher mean channel SNR/symbol ( $>15 \mathrm{~dB}$ ), the SNR penalty for 16-PSK with respect to 16-QAM is approximately 5 dB. Fig. 7, Fig. 8 and Fig. 10 also highlight the utility and versatility of our proposed analytical technique to yield an accurate performance prediction of CAF relay networks in a Rice fading environment, which hitherto had resisted a simple analytical solution. For example, we could not directly evaluate the desired outage probability using [38, Eq. (7) or (8)] in conjunction with (23) since most mathematical software packages such as MATLAB and MATHEMATICA do not have a built-in function for the generalized Marcum Q-function with complex arguments. But our approximate MGF formula does not contain any special mathematical functions or pose similar restrictions, and thus it may be readily used with [38, Eqs. (7) - (8)] to compute the desired symbol error outage.

\section{CONCLUDING REMARKS}

In this article, we have developed an accurate exponential-type approximation for the CEP of a broad class of coherent, differentially coherent, and non-coherent digital modulation schemes and a novel asymptotic MGF formula for the end-to-end SNR in a CAF relayed path over generalized fading environments with i.n.d fading statistics. We also develop a unified expression for the ASER of CAF relay networks (i.e., Eq. (13)) in closed-form. In particular, our MGF-based approximation framework yields considerably better predictions of the actual ASER compared to the modified RCG method at the low-SNR regime and at higher values of fading severity indices, while being easier to program and evaluate when compared to the existing bounding techniques or exact expressions (when available). Moreover, our simple and elegant closed-form ASER formula may be further exploited for system-level optimization such as optimal power allocation problem in cooperative relay networks and link-adaptive CAF wireless network design. These extensions along with the asymptotic analysis of multi-hop multi-relay CAF networks will be pursued as part of our future work.

\section{ACKNOWLEDGEMENTS}

This work was supported in part by funding from the US Army Research Office (W911NF-042-0054), Clarkson Aerospace, and the National Science Foundation (0931679 and 1040207). 
International Journal of Wireless \& Mobile Networks (IJWMN) Vol. 4, No. 1, February 2012

\section{REFERENCES}

[1] M. O. Hasna and M. S. Alouini, (2003) "End-to-End Performance of Transmission Systems with Relays over Rayleigh-Fading Channels," IEEE Trans. Wireless Commun., vol. 2, pp. 1126-1131.

[2] P. A. Anghel and M. Kaveh, (2004) "Exact Symbol Error Probability of a Cooperative Network in a Rayleigh-Fading Environment," IEEE Trans. Wireless Communications, vol. 3, pp. 1416-1421.

[3] W. Su, K. S. Ahmed and K. J. Ray Liu, (2008) "Cooperative Communication Protocols in Wireless Networks: Performance Analysis and Optimum Power Allocation,” Springer Journal on Wireless Personal Communications, vol. 44, pp. 181-217.

[4] M. O. Hasna and M. S. Alouini, (2004) "Harmonic Mean and End-to-End Performance of Transmission Systems With Relays,” IEEE Trans. Communications, vol. 52, pp. 130-135.

[5] A Ribeiro, X. Cai and G. Giannakis, (2005) "Symbol Error Probabilities for General Cooperative Links," IEEE Trans. Wireless Communications, vol. 4, pp. 1264-1273.

[6] Z. Wang and G. Giannakis, (2003) "A Simple and General Parameterization Quantifying Performance in Fading Channels,” IEEE Trans. Communications, vol. 51, pp. 1389-1398.

[7] Y. Ma, Z. Wang and S. Pasupathy, (2003) "Asymptotic Performance of Wireless Communications with Generalized Selection Combining,” Proc. IEEE GLOBECOM'03, pp. 1679-1683.

[8] T. Nechiporenko, K. Phan, C. Tellambura and H. Nguyen, (2009) "Capacity of Rayleigh Fading Cooperative Systems under Adaptive Transmission," IEEE Trans. Wireless Communications, vol. 8, pp. 1626-1631.

[9] S. Ikki and M. Ahmed, (2007) "Performance Analysis of Cooperative Diversity Wireless Networks over Nakagami-m Fading Channels,” IEEE Communications Letters, vol. 11, pp. 334-336.

[10] A. Annamalai, B. Modi, R. Palat and J. Matyjas, (2010) "Tight Bounds on the Ergodic Capacity of Cooperative Analog Relaying with Adaptive Source Transmission Techniques," Proc. IEEE PIMRC'10, Istanbul, pp. 18-23.

[11] B. C. Modi, O. Olabiyi and A. Annamalai, (2011) "On Ergodic Capacity of Cooperative Amplifyand-Forward Relay Networks in Rice Fading Environments," Proc. IEEE GLOBECOM Workshop HeterWMN'11, Houston.

[12] N. Laneman, D. Tse and G. Wornell, (2004) "Cooperative Diversity in Wireless Networks: Efficient Protocols and Outage Behaviour,” IEEE Trans. Info. Theory, vol. 50, pp. 3062-3080.

[13] M. K. Simon and M. S. Alouini, (2005) Digital Communication over Fading Channels, $2^{\text {nd }}$ ed., John Wiley \& Sons.

[14] B. Maham and A. Hjørungnes, (2009) "Asymptotic Performance Analysis of Amplify-and-Forward Cooperative Networks in a Nakagami-m Fading Environment," IEEE Communications Letters, vol. 13, pp. 300-302.

[15] M. Di Renzo, F. Graziosi and F. Santucci, (2009) “A Unified Framework for Performance Analysis of CSI-assisted Cooperative Communications over Fading Channels," IEEE Trans. Communications, vol. 57, pp. 2551-2557.

[16] J. Laneman and G. Wornell, (2003) "Distributed Space-Time Coded Protocols for Exploiting Cooperative Diversity in Wireless Networks," IEEE Trans. Info. Theory, vol. 49, pp. 2415-2525.

[17] A. Sendonaris, E. Erkip and B. Aazhang, (2003) "User Cooperation Diversity, Part I: System Description," IEEE Trans. Communications, vol. 51, pp. 1927-1938.

[18] A. Sendonaris, E. Erkip and B. Aazhang, (2003) "User Cooperation Diversity, Part II: Implementation Aspects and Performance Analysis," IEEE Trans. Communications, vol. 51, pp. 1939-1948.

[19] M. Khojastepour, A. Sabharwal and B. Aazhang, (2004) "Lower Bounds on the Capacity of Gaussian Relay Channel,” Proc. CISS'04, Princeton, pp. 597-602. 
International Journal of Wireless \& Mobile Networks (IJWMN) Vol. 4, No. 1, February 2012

[20] A. Bletsas, A. Khisti, D. P. Reed and A. Lippman, (2006) "A Simple Cooperative Diversity Method Based on Network Path Selection,” IEEE J. Selected Areas Commun., vol. 24, pp. 659-672.

[21] W. Su, A. K. Sadek and K. J. R. Liu, (2005) "SER Performance Analysis and Optimum Power Allocation for Decode-and-Forward Cooperation Protocol in Wireless Networks," Proc. IEEE WCNC'05, pp. 984-989.

[22] A. K. Sadek, W. Su and K. J. R. Liu, (2005) "Performance Analysis for Multi-Node Decode-andForward Relaying in Cooperative Wireless Networks," Proc. IEEE ICASSP'05, pp. 521-524.

[23] Y. Lee and M. H. Tsai, (2009) "Performance of Decode-and-Forward Cooperative Communications over Nakagami-m Fading Channels,” IEEE Trans. Vehicular Technology, vol. 58, pp. 1218-1228.

[24] N. Beaulieu and J. Hu, (2006) "A Closed-Form Expression for the Outage Probability of Decodeand-Forward Relaying in Dissimilar Rayleigh Fading Channels," IEEE Communications Letters, vol. 10, pp.813-815.

[25] S. Ikki and M. H. Ahmed, (2010) "Performance Analysis of Incremental-Relaying Cooperative Diversity Networks over Rayleigh Fading Channels," IET Communications, vol. 5, pp. 337-349.

[26] Y. G. Kim and N. C. Beaulieu, (2011) "Exact Closed-Form Solutions for the BEP of Decode-andForward Cooperative Systems in Nakagami-m Fading Channels," IEEE Trans. Communications, vol. 59 , pp. $2355-2361$.

[27] Y. Hu, K. H. Li and K. C. Teh, (2009) "Performance and Diversity Analysis of Decode-andForward Cooperative System over Nakagami-m Fading Channels," Wiley Journal on Wireless Communications and Mobile Computing, vol. 11, pp. 742-749.

[28] R. Nikjah and N. C. Beaulieu, (2011) "On the Average Capacity of Rate Adaptive Single-Relay Selection Decode-and-Forward Dual-Hop Relaying in Rayleigh Fading Channels," Proc. IEEE WCNC'11, pp. $1730-1735$.

[29] T. Nechiporenko, P. Kalansuriya and C. Tellambura, (2009) "Performance of Optimum Switching Adaptive M-QAM for Amplify-and-Forward Relays," IEEE Trans. Vehicular Technology, vol. 58, pp. 2258-2268.

[30] T. Nechiporenko, K. Phan, C. Tellambura and H. Nguyen, (2008) "Performance Analysis of Adaptive M-QAM for Rayleigh Fading Cooperative Systems,' Proc. IEEE ICC'08, pp. 3393-3399.

[31] B. Modi and A. Annamalai, (2011) "Improving the Spectral Efficiency of Amplify-and-Forward Cooperative Relay Network with Adaptive M-QAM Modulation,” Proc. IEEE ICCCN'11, Maui.

[32] A. Annamalai, B. Modi and R. C. Palat, (2011)“Analysis of Cooperative Non-regenerative Relay Networks with Adaptive Modulation in Generalized Fading Channels," Proc. IEEE IWCMC'11, Istanbul, pp. 760-765.

[33] B. Modi, O. Olabiyi and A. Annamalai, (2011) "Improving the Spectral Efficiency of Adaptive Modulation in Amplify-and-Forward Cooperative Relay Networks with Truncated ARQ Protocol," Proc. IEEE GLOBECOM'11, Houston.

[34] B. Modi, O. Olabiyi, and A. Annamalai, (2011) "Joint-Design of Adaptive Modulation and Coding with ARQ for Cooperative Relay Networks,” Proc. IEEE MILCOM'11, Baltimore, pp. 67-72.

[35] I. S. Gradshteyn and I. M. Ryzhik, (1994) Table of Integrals, Series and Products, $5^{\text {th }}$ ed., San Diego: Academic Press.

[36] A. Annamalai and C. Tellambura, (2002) "Analysis of Hybrid Selection/Maximal-Ratio Diversity Combiners With Gaussian Errors,” IEEE Trans. Wireless Communications, vol. 1, pp. 498-511.

[37] O. Olabiyi and A. Annamalai, (2011) "ASER Analysis of Cooperative Non-Regenerative Relay Systems over Generalized Fading Channels," Proc. IEEE ICCCN'11, Maui.

[38] R. Palat, A. Annamalai and J. Reed, (2008) "An Efficient Method for Evaluating Information Outage Probability and Ergodic Capacity of OSTBC Systems," IEEE Communications Letters, vol. 12, pp. 191-193. 
Oluwatobi O. Olabiyi received the B.Sc. degree in Electronic and Electrical Engineering from Obafemi Awolowo University, Ile-Ife, Nigeria, and the M.S. degree in Electrical Engineering Prairie View A\&M University, Texas, USA. Over the last two years, he has co-authored approximately two-dozen peer-reviewed conference and journal articles. He was the recipient of the Roy G. Perry College of Engineering Outstanding Masters Student of the Year Award (2011) and the National Society of Black Engineer's Golden Torch Award for Graduate Student of Year (2012). He is presently continuing his doctoral studies at the Prairie View A\&M University. His research interests include dynamic spectrum access, MIMO, cooperative communications, statistical signal processing, compressive sensing, machine-learning and optimization techniques.

Dr. Annamalai is presently the Director of Center of Excellence for Communication Systems Technology Research, a Texas A\&M Board of Regents approved University Research Center at the Prairie View A\&M University, and a tenured faculty member in the Department of Electrical and Computer Engineering. He has over 16 years of research/teaching experience in wireless communications at Motorola, University of Victoria, Air Force Research Laboratory, Virginia Tech and PVAMU with approximately 200 peer-reviewed publications and 5 book chapters. Dr. Annamalai has been honored by his colleagues on numerous occasions for his excellence in research including winning the 2011 Roy G. Perry College of Engineering

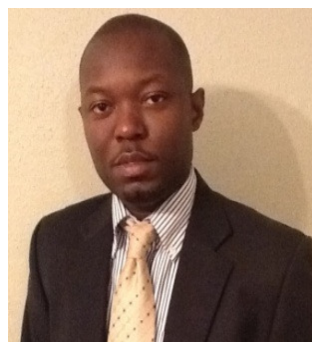
Outstanding Faculty (Research) Award, IEEE Leon Kirchmayer Prize Paper award, ASEE/AFOSR Summer Faculty Fellowships, NSERC Doctoral Prize, CAGS/UMI Distinguished Doctoral Dissertation Award, IEEE VTS/Motorola Daniel E. Noble Fellowship, among others. He had served on the Editorial Boards of four IEEE journals/transactions in the last 12 years, and has helped to organize a few major IEEE conferences on wireless communications including serving in the capacity of Technical Program Chair of the 2002 IEEE Vehicular Technology Conference in Vancouver, Canada. His current research interests include cooperative spectrum sensing, compressive sensing, cross-layer design for scalable multimedia transmission and cooperative wireless communications. 